\title{
Resonantly damped oscillations of longitudinally stratified coronal loops
}

\author{
M. V. Dymova and M. S. Ruderman
}

\begin{abstract}
Department of Applied Mathematics, University of Sheffield, Hicks Building, Hounsfield Road, Sheffield S3 7RH, UK e-mail: M.S.Ruderman@sheffield.ac.uk
\end{abstract}

Received 20 February 2006 / Accepted 12 July 2006

ABSTRACT

\begin{abstract}
Soon after coronal loop oscillations were observed by TRACE spacecraft for the first time in 1999, various theoretical models have been put forward to explain the rapid damping of the oscillations of these intriguing objects. Coronal loop oscillations are often interpreted as fast kink modes of a straight cylindrical magnetic flux tube with immovable edges modelling dense photospheric plasma at the ends of the loop. Taking this model as a basis we use cold plasma approximation and consider the tube to be thin to simplify the problem and be able to deal with it analytically. In its equilibrium state the tube is permeated by a homogeneous magnetic field directed along the tube axis. We include the effect of stratification in our model supposing that plasma density varies along the tube. There is also density inhomogeneity in the radial direction confined in a layer with thickness much smaller than the radius of the tube. Considering the system of linearized MHD equations we study the dependence of the spectrum of tube oscillations and its damping due to resonant absorption on the parameters of the unperturbed state. The implication of the obtained results on coronal seismology is discussed.
\end{abstract}

Key words. magnetohydrodynamics (MHD) - waves - plasmas

\section{Introduction}

TRACE spacecraft has observed transverse coronal loop oscillations for several years (e.g., Aschwanden et al. 1999, 2002; Nakariakov et al. 1999; Schrijver \& Brown 2000; Schrijver et al. 2002). These oscillations were interpreted by Nakariakov et al. (1999) as fast kink modes of magnetic flux tubes. The observations revealed that the transverse coronal loop oscillations are very strongly damped with the oscillation period ranging from 2.3 to $10.8 \mathrm{~min}$ and the oscillations decay time from 3.2 to $20.8 \mathrm{~min}$. The rapid damping of the oscillations (within several periods) has been a subject for a number of studies and different mechanisms of damping were proposed (see. e.g., Roberts 2000; Ruderman 2005). At present the most reliable mechanism of damping of transverse coronal loop oscillations is the energy resonant absorption due to coupling between the global loop oscillation and the local Alfvén oscillations. To our knowledge Hollweg \& Yang (1988) were the first who suggested that the coronal loop oscillations can damp very efficiently due to resonant absorption. Goossens et al. (1992) thoroughly studied this damping and, in particular, obtained an analytical expression for the damping rate of the kink mode of a straight non-stratified magnetic tube in the case when the density varies in the radial direction only in a thin layer near the tube boundary.

The observation of damped transverse oscillations of coronal magnetic loops strongly boosted studies of damping of magnetic tube oscillations due to resonant absorption. Ruderman \& Roberts (2002) analytically solved the initial value problem for a straight unstratified magnetic tube under assumption that the radial density variation occurs only in a thin layer of thickness $l$ near the tube boundary. They showed that, after the time of order of a few periods of the tube oscillation a damped kink mode of the tube is formed. After that this mode starts to damp with the damping rate proportional to $l / R \ll 1$, where $R$ is the tube radius. They applied their theoretical results to the observational data reported by Nakariakov et al. (1999), and found that the observed damping rate corresponds to $l / R \approx 0.23$. Recently Terradas et al. (2006) solved the same problem as Ruderman \& Roberts (2002) numerically for arbitrary $l / R$. In particular, they found that a damped kink mode of the tube is formed even faster than the analytic theory predicts, usually within one or two periods of oscillations.

Goossens et al. (2002) applied the expression for the resonant damping rate to investigate the damped coronal loop oscillations in a sample of 11 loops provided by Aschwanden et al. (2002). They found that the ratio $l / R$ corresponding to the observed damping rates varies from 0.16 to 0.49 . Hence, at least for some events, $l / R$ is not small at all. This observation inspired Van Doorsselaere et al. (2004a) to calculate the resonant damping rate numerically for arbitrary $l / R$. They found that the analytical expression gives a very good approximation of the damping rate for $l / R \leq 0.4$. However, for larger values of $l / R$ there is substantial difference between the analytical and numerical results. When $l / R$ takes its maximum value, $l / R=1$, this difference is equal to $25 \%$. (Note that Van Doorsselaere et al. (2004a) used the definition of the tube radius, $\widetilde{R}$, different from that used by Ruderman \& Roberts (2002). The relation between the two definitions is $\widetilde{R}=R-l / 2$.) The results obtained by Van Doorsselaere et al. (2004a), in particular, show that the approximation of small $l / R$ works very well for 8 events out of 11 considered by Goossens et al. (2002), and reasonably well for 3 remaining events.

Further progress in studying the damped transverse oscillations of coronal loops is related to considering more complicated models of the loops. Ruderman (2003) considered 
resonantly damped kink oscillations of a magnetic tube with the elliptic cross-section. Van Doorsselaere et al. (2004b) studied the damped oscillations of a thin magnetic tube of a semi-circular shape to investigate the curvature effect.

Observations revealed that "All loop oscillations occur in relatively high loops, with loop top heights exceeding approximately $60 \mathrm{Mm}$ and up to $150 \mathrm{Mm}$ " (Schrijver et al. 2002). Hence the loop heights are comparable with the density scale height in the corona. This observation motivated Andries et al. (2005a) to study the effect of stratification on the damped transverse oscillations of coronal loops. In their study Andries et al. modelled the loop as a straight magnetic tube with the density varying both along the tube and in the radial direction, and assumed $l / R \ll 1$. Then they used the regular perturbation method with $l / R$ as a small parameter. In the first order approximation they calculated the (real) frequency of the tube kink oscillation, and in the second order approximation they found the damping rate due to resonant absorption. The frequencies of the fundamental mode and overtones are determined by the condition that an infinite determinant with the elements nonlinearly dependent on the frequency $\omega$ is equal to zero. To calculate $\omega$ Andries et al. truncated the determinant thus obtaining a complicated nonlinear transcendent equation for $\omega$. Then they solved this equation numerically, which was an involved numerical procedure.

The approach used by Andries et al. (2005a) works for arbitrary ratio of the tube radius $R$ to its half-length $L$. However observations show that this ratio is small. Definitely $R / L<0.1$, with the typical values between 0.03 and 0.07 . This implies that $R / L$ can be used as a small parameter, which substantially simplifies the analysis. The aim of this paper is to develop the asymptotic theory of damped kink oscillations of stratified magnetic loops valid for $R / L \ll 1$.

The paper is organized as follows. In the next section we describe main assumptions used in our model and derive equations governing the plasma motion. In Sect. 3 we obtain the solution of the governing equations in internal and external homogeneous regions, where the plasma density varies in the longitudinal direction but does not vary in the radial direction. In Sect. 4 we discuss the two-dimensional extension of connection formulae previously obtained for one-dimensional equilibria. In Sect. 5 we calculate the variations of the radial velocity and magnetic pressure across the region where the density varies in the radial direction. In Sect. 6 we match the solutions in the internal and external regions, thus obtaining the Sturm-Liouville problem determining the oscillation frequency, and the expression for the damping rate. In Sect. 7 we calculate the frequencies and decrements of the fundamental mode and first overtone for a particular density distribution, and discuss the application of the obtained results to coronal seismology. Section 8 contains the summary and our conclusions.

\section{Basic assumptions and governing equations}

We model a solar coronal loop by a straight axisymmetric magnetic flux tube. Figure 1 shows the sketch of the equilibrium state. In what follows we use cylindrical coordinates $r, \varphi, z$. The tube length is $2 L$, and its radius $R$. The plasma density, $\rho(r, z)$, outside the tube $(r>R)$ is equal to $\rho_{\mathrm{e}}(z)$. Inside the tube the plasma density varies in the radial direction only in the annulus $R-l<r<R$, so that $\rho(r, z)=\rho_{\mathrm{i}}(z)$ for $r<R-l$. Stratification is assumed to be everywhere the same: $\rho_{\mathrm{e}}(z) / \rho_{\mathrm{i}}(z)=\chi<1$, where $\chi$ is a constant. We assume that the dependence on $r$ in the annulus can be factored out and take $\rho(z, r)=f(r) \rho_{\mathrm{i}}(z)$ for $R-l<r<R$. In what follows we will call this assumption the

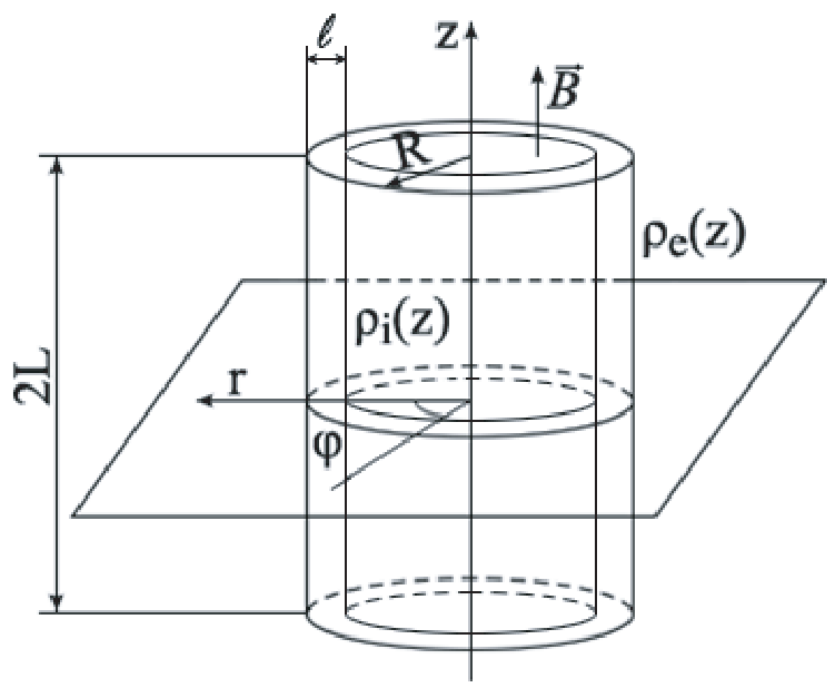

Fig. 1. The sketch of the equilibrium state. The ends of the magnetic tube are assumed to be frozen in a dense photospheric plasma.

assumption of uniform stratification. The density is assumed to be continuous, so that $f(R-l)=1$ and $f(R)=\chi$. We also assume that the density varies monotonically, so that $f(r)$ is a monotonically decreasing function. The plasma is permeated by a uniform magnetic field $\boldsymbol{B}$ directed along the tube axis, $\boldsymbol{B}=B \hat{z}$, where $\hat{z}$ is the unit vector along the $z$-axis. The magnetic field lines are anchored in the photosphere, so that the footpoints are subject to the line-tying conditions.

In what follows we use the cold plasma approximation and neglect the plasma pressure in comparison with the magnetic pressure. We assume that $R \ll L$ and consider oscillations with the wavelength of order $L$. The only dissipative effect that we take into account is the plasma viscosity. In accordance with the classical Braginskii's expression for the viscosity tensor in a magnetized plasma (Braginskii 1965), under typical coronal conditions, the shear viscosity is at least 10 orders of magnitude smaller than the compressional viscosity. However, in the problem of coronal loop oscillations, dissipation is only important in the Alfvén dissipative layer embracing an ideal resonant surface. Ofman et al. (1994) and Erdélyi \& Goossens (1995) showed that in Alfvén dissipative layers only the shear viscosity is significant, while all other terms in Braginskii's expression for the viscosity tensor can be neglected. In accordance with this fact we write the viscous force in the momentum equation in a simplified form $\rho v \nabla^{2} v$, where $v$ is the velocity and $v$ the kinematic viscosity. In the dissipative layer, there are large gradients in the radial direction only. This observation enables us to use the approximation $\nabla^{2} v \approx \partial^{2} v / \partial r^{2}$. Then the system of linear MHD equations for a cold plasma takes the form

$$
\begin{aligned}
& \frac{\partial u}{\partial t}=\frac{1}{\rho}\left(\frac{B}{\mu_{0}} \frac{\partial b_{r}}{\partial z}-\frac{\partial P}{\partial r}\right)+v \frac{\partial^{2} u}{\partial r^{2}} \\
& \frac{\partial w}{\partial t}=\frac{1}{\rho}\left(\frac{B}{\mu_{0}} \frac{\partial b_{\varphi}}{\partial z}-\frac{1}{r} \frac{\partial P}{\partial \varphi}\right)+v \frac{\partial^{2} w}{\partial r^{2}} \\
& \frac{\partial b_{r}}{\partial t}=B \frac{\partial u}{\partial z}, \quad \frac{\partial b_{\varphi}}{\partial t}=B \frac{\partial w}{\partial z} \\
& \frac{\partial P}{\partial t}=-\frac{B^{2}}{r \mu_{0}}\left(\frac{\partial(r u)}{\partial r}+\frac{\partial w}{\partial \varphi}\right)
\end{aligned}
$$


$\frac{1}{r} \frac{\partial\left(r b_{r}\right)}{\partial r}+\frac{1}{r} \frac{\partial b_{\varphi}}{\partial \varphi}+\frac{\partial b_{z}}{\partial z}=0$.

Here $\boldsymbol{v}=(u, w, 0)$ is the velocity, $\boldsymbol{B}=\left(b_{r}, b_{\varphi}, b_{z}+B\right)$ the magnetic field, $P=B b_{z} / \mu_{0}$ the perturbation of the magnetic pressure, and $\mu_{0}$ the magnetic permeability of free space. Since the equilibrium quantities depend on $r$ and $z$ coordinates only, we can Fourier-analyze the perturbed quantities with respect to $\varphi$ and $t$ and take them proportional to $\exp (-\mathrm{i} \omega t+\mathrm{i} m \varphi)$, where $m$ (a positive integer) is the azimuthal wave number, and $\omega$ is the frequency of oscillations. In what follows we consider the kink mode of tube oscillations only and take $m=1$.

Using Eqs. (3) to eliminate $b_{r}$ and $b_{\varphi}$ from Eqs. (1) and (2) we obtain

$\frac{\partial^{2} u}{\partial z^{2}}+\frac{\omega^{2}}{V_{\mathrm{A}}^{2}} u-\frac{\mathrm{i} v \omega}{V_{\mathrm{A}}^{2}} \frac{\partial^{2} u}{\partial r^{2}}=-\frac{\mathrm{i} \omega}{\rho V_{\mathrm{A}}^{2}} \frac{\partial P}{\partial r}$,

$\frac{\partial^{2} w}{\partial z^{2}}+\frac{\omega^{2}}{V_{\mathrm{A}}^{2}} w-\frac{\mathrm{i} v \omega}{V_{\mathrm{A}}^{2}} \frac{\partial^{2} w}{\partial r^{2}}=\frac{\omega}{r \rho V_{\mathrm{A}}^{2}} P$,

where $V_{\mathrm{A}}(r, z)=B\left[\mu_{0} \rho(r, z)\right]^{-1 / 2}$ is the Alfvén speed.

The system of Eqs. (6) and (7) is not closed: there are three variables, $u, w$ and $P$, and only two equations, so that we need one additional equation. It turns out that it is convenient to use different equations outside and inside the inhomogeneous annulus, $R-l<r<R$, to close the system (6) and (7). We start from deriving the third equation that will be used outside the annulus. When doing so we keep in mind that, outside the annulus, $\rho=\rho(z)$, and we can take $v=0$ since viscous effects are only significant in the dissipative layer. Now we multiply Eq. (1) by $r \rho$ and differentiate the result with respect to $r$. Then we multiply Eq. (2) by $\rho$ and differentiate the result with respect to $\varphi$. Adding the obtained equations, using Eqs. (4) and (5), and recalling that perturbations of all variables are proportional to $\exp (-\mathrm{i} \omega t+\mathrm{i} \varphi)$, we arrive at

$\frac{\partial^{2} P}{\partial z^{2}}+\frac{1}{r} \frac{\partial}{\partial r}\left(r \frac{\partial P}{\partial r}\right)+\left(\frac{\omega^{2}}{V_{\mathrm{A}}^{2}}-\frac{1}{r^{2}}\right) P=0$.

The reason why we do not use Eq. (8) and need another equation in the annulus is the following. To derive the equation governing the tube oscillations we need to calculate the variation of $u$ and $P$ across the annulus. The variation of $P$ is determined by Eq. (6). To determine the variation of $u$ we need an equation containing the derivatives of $u$ with respect to $r$ on the left-hand side, and not containing the derivatives with respect to $r$ on the right-hand side. Now we proceed to the derivation of such an equation. We use Eq. (4) to express $w$ in terms of $u$ and $P$. Substituting the result into Eq. (7) we obtain

$$
\begin{aligned}
\frac{\partial^{3}(r u)}{\partial r \partial z^{2}}+\frac{\omega^{2}}{V_{\mathrm{A}}^{2}} \frac{\partial(r u)}{\partial r}- & \frac{\mathrm{i} v \omega}{V_{\mathrm{A}}^{2}} \frac{\partial^{3}(r u)}{\partial r^{3}} \\
& =\frac{\mathrm{i} r \omega}{\rho V_{\mathrm{A}}^{2}}\left[\frac{\partial^{2} P}{\partial z^{2}}+\left(\frac{\omega^{2}}{V_{\mathrm{A}}^{2}}-\frac{1}{r^{2}}\right) P\right] .
\end{aligned}
$$

When deriving Eq. (9), we have neglected terms proportional to $v \partial^{2} P / \partial r^{2}$. This can be done for the following reason. Viscosity is only important in the dissipative layer where there are large gradients of perturbations. However, we will see in what follows that there are no large gradients of $P$ in the dissipative layer.

To complete the formulation of the problem, we have to specify the boundary conditions. At the ends of the tube the linetying conditions have to be satisfied:

$u=w=0 \quad$ at $\quad z= \pm L$.
It follows from Eqs. (4) and (10) that

$P=0 \quad$ at $\quad z= \pm L$.

Finally, we consider only non-leaky modes of the tube oscillations, so that all perturbations have to decay as $r \rightarrow \infty$.

When the tube is not stratified in the longitudinal direction, i.e. all the equilibrium quantities depend on $r$ only, we Fourieranalyze $u, w$ and $P$ and take them proportional to $\cos (\pi n z / 2 L)$ with integer $n$ (e.g., Ruderman \& Roberts 2002), thus reducing Eqs. (6)-(9) to ordinary differential equations. However it is not possible when the loop is longitudinally stratified because in that case the coefficients of Eqs. (6)-(9) depend both on $r$ and $z$. To overcome this difficulty we use generalized Fourier expansions with respect to eigenfunctions of the following Sturm-Liouville problem:

$V_{\mathrm{A}}^{2} \frac{\mathrm{d}^{2} W}{\mathrm{~d} z^{2}}=-\lambda W, \quad W( \pm L)=0$.

The eigenvalues of this problem are real, and they constitute a monotonically growing sequence $\left\{\lambda_{n}\right\}, \lambda_{n} \rightarrow \infty$ as $n \rightarrow \infty$ (e.g. Coddington \& Levinson 1955). It is easy to show that all the eigenvalues are positive. Any square integrable in the interval $[-L, L]$ function $g(z)$ can be expanded in the generalized Fourier series

$g(z)=\sum_{n=1}^{\infty} g_{n} W_{n}(z)$

where $W_{n}(z)$ is an eigenfunction of the Sturm-Liouville problem (12) corresponding to the eigenvalue $\lambda_{n}$. Obviously, they can be chosen to be real. The eigenfunctions satisfy the orthogonality condition

$\int_{-L}^{L} V_{\mathrm{A}}^{-2}(z) W_{n}(z) W_{m}(z) \mathrm{d} z=0$ for $m \neq n$.

If $g(z)$ has a continuous second derivative and satisfies the boundary conditions $g( \pm L)=0$, then the sum in (13) is uniformly convergent, and (13) can be differentiated twice (e.g. Titchmarsh 1946; Naimark 1967). Note that, since $V_{\mathrm{A}}$ and $g$ depend also on $r, \lambda_{n}, W_{n}$ and $g_{n}$ depend on $r$ as on a parameter. Since we assume that $\rho(r, z)=f(r) \rho_{\mathrm{i}}(z)$, we can rewrite Eq. (12) as

$V_{\mathrm{Ai}}^{2} \frac{\mathrm{d}^{2} W}{\mathrm{~d} z^{2}}=-\lambda f(r) W, \quad W( \pm L)=0$

Then it is obvious that $W_{n}$ can be chosen to be independent of $r$, while the dependence of $\lambda_{n}$ on $r$ is given by

$\lambda_{n}(r)=\frac{\lambda_{n}(R-l)}{f(r)}$

For convenience we also assume that $W_{n}(z)$ is normalized by the condition

$\int_{-L}^{L} V_{\mathrm{Ai}}^{-2}(z) W_{n}(z)^{2} \mathrm{~d} z=1$,

so that the Fourier coefficients are given by

$g_{n}=\int_{-L}^{L} V_{\mathrm{Ai}}^{-2}(z) g(z) W_{n}(z) \mathrm{d} z$. 


\section{Solutions in the homogeneous regions}

In this section we obtain solutions for $P$ and $u$ in the homogeneous regions, where $\rho$ depends on $z$ only. These are the internal region, determined by the inequality $r<R-l$, and the external region, determined by the inequality $r>R$. In what follows we use the approach applied by Dymova \& Ruderman (2005) to studying kink oscillations of stratified magnetic tubes homogeneous in the radial direction. In the homogeneous regions we use Eq. (6) with the third term on the left-hand side equal to zero, and Eq. (8). We start our analysis from the internal region. In this region the characteristic scale in the $r$-direction is $R$, while the characteristic scale in the $z$-direction is $L$. This implies that the ratios of the first term and the term proportional to $\omega^{2}$ on the lefthand side of Eq. (8) to the second term is of the order $(R / L)^{2}$. Since $R / L \ll 1$, the first term and the term proportional to $\omega^{2}$ can be neglected and Eq. (8) reduces to

$r \frac{\partial}{\partial r}\left(r \frac{\partial P}{\partial r}\right)-P=0$.

The solution of this equation regular at $r=0$ is

$P(r, z)=r A_{\mathrm{i}}(z)$,

where $A_{\mathrm{i}}(z)$ is an arbitrary function satisfying $A_{\mathrm{i}}( \pm L)=0$.

Now we expand $u$ and $P$ in the generalized Fourier series of the form (13). Substituting these expansions in Eq. (6) with the third term on the left-hand side equal to zero, we obtain

$u_{n}=\frac{\mathrm{i} \omega \Phi_{n}}{\lambda_{n}-\omega^{2}}$,

where $\Phi_{n}$ is the $n$th Fourier coefficient of function $\Phi(z)=$ $A_{\mathrm{i}}(z) / \rho_{\mathrm{i}}(z)$. Note that $u_{n}$ is independent of $r$, so that $u$ in the internal region is a function of $z$ only.

In the external region the characteristic scales in the $r$ and $z$-direction are the same and equal to $L$, so that we cannot neglect any terms on the left-hand side of (8). To make analytical progress, we assume in what follows that the inhomogeneous annulus is thin, i.e. $l \ll R$. Then we use the regular perturbation method with $l / R$ as a small parameter. We will see in what follows that the ratio of the imaginary part of $\omega$ to its real part is of the order $l / R$, so that we can write $\omega=\omega_{r}+\mathrm{i}(l / R) \omega_{i}$, where $\omega_{r}$ and $\omega_{i}$ are real and $\omega_{i} \sim \omega_{r}$.

Let us now look for the solution to Eq. (8) in the form of expansion $P=P^{(0)}+(l / R) P^{(1)}+\ldots$ Then, in the zero order approximation, we obtain

$\frac{\partial^{2} P^{(0)}}{\partial z^{2}}+\frac{1}{r} \frac{\partial}{\partial r}\left(r \frac{\partial P^{(0)}}{\partial r}\right)+\left(\frac{\omega_{r}^{2}}{V_{\mathrm{Ae}}^{2}}-\frac{1}{r^{2}}\right) P^{(0)}=0$.

We look for the solution to this equation in the form $P^{(0)}(r, z)=$ $F^{(0)}(r) G(z)$. Separating the variables we obtain that $F^{(0)}(r)$ satisfies the equation

$\frac{\mathrm{d}^{2} F^{(0)}}{\mathrm{d} r^{2}}+\frac{1}{r} \frac{\mathrm{d} F^{(0)}}{\mathrm{d} r}-\left(\Lambda^{2}+\frac{1}{r^{2}}\right) F^{(0)}=0$,

while $G(z)$ is a solution of the Sturm-Liouville problem

$\frac{\mathrm{d}^{2} G}{\mathrm{~d} z^{2}}+\frac{\omega_{r}^{2}}{V_{\mathrm{Ae}}^{2}} G=-\Lambda^{2} G, \quad G=0$ at $z= \pm L$,

where $\Lambda^{2}$ is the separation constant. The eigenvalues of the Sturm-Liouville problem (24) constitute a monotonically increasing sequence $\left\{\Lambda_{n}^{2}\right\}, n=1,2, \ldots$, such that $\Lambda_{n}^{2} \rightarrow \infty$ as $n \rightarrow \infty$ (e.g. Coddington \& Levinson 1955). The eigenfunctions $G_{n}$ corresponding to the eigenvalues $\Lambda_{n}^{2}$ constitute a complete set of functions in the space of square integrable functions, so that any such function can be expanded in a generalized Fourier series with respect to functions $G_{n}$, the series being convergent in the quadratic mean. If a function has continuous second derivative and is equal to zero at $z= \pm L$, then the corresponding generalized Fourier series converges uniformly with respect to $z$ and can be differentiated twice (e.g. Titchmarsh 1946; Naimark 1967).

It is explained in Dymova \& Ruderman (2005) that the case $\Lambda_{1}^{2}<0$ corresponds to a leaky mode. We assume a priori that there are no leaky modes, so that $\Lambda_{1}^{2}>0$. We will verify this assumption after we find $\omega$. Since the sequence $\left\{\Lambda_{n}^{2}\right\}$ is monotonically increasing, the assumption $\Lambda_{1}^{2}>0$ implies that $\Lambda_{n}^{2}>0$ for any $n$. Then Eq. (23) is the modified Bessel equation and, with the accuracy up to a multiplicative constant, its solution decaying at infinity is $F(r)=K_{1}\left(\Lambda_{n} r\right)$, where $K_{1}$ is the modified Bessel function of the second kind (McDonald function). Then, due to the expansion theorem (e.g. Titchmarsh 1946; Naimark 1967), the general solution of Eq. (22) is given by

$P^{(0)}=\sum_{n=1}^{\infty} H_{n}^{(0)} G_{n}(z) K_{1}\left(\Lambda_{n} r\right)$

where $H_{n}^{(0)}$ are arbitrary constants.

In the first order approximation we obtain from Eq. (8)

$\frac{\partial^{2} P^{(1)}}{\partial z^{2}}+\frac{1}{r} \frac{\partial}{\partial r}\left(r \frac{\partial P^{(1)}}{\partial r}\right)+\left(\frac{\omega_{r}^{2}}{V_{\mathrm{Ae}}^{2}}-\frac{1}{r^{2}}\right) P^{(1)}=-\frac{2 \mathrm{i} \omega_{r} \omega_{i}}{V_{\mathrm{Ae}}^{2}} P^{(0)}$.

Let us now look for the solution to this equation in the form of expansion

$P^{(1)}=\sum_{n=1}^{\infty} F_{n}^{(1)} G_{n}(z)$.

Substituting (27) in (26), using (24) and (25), and taking into account that $G_{n}(z)$ are linearly independent functions, we obtain

$\frac{\mathrm{d}^{2} F_{n}^{(1)}}{\mathrm{d} r^{2}}+\frac{1}{r} \frac{\mathrm{d} F_{n}^{(1)}}{\mathrm{d} r}-\left(\Lambda_{n}^{2}+\frac{1}{r^{2}}\right) F_{n}^{(1)}=-\frac{2 \mathrm{i} \omega_{r} \omega_{i}}{V_{\mathrm{Ae}}^{2}} H_{n}^{(0)} K_{1}\left(\Lambda_{n} r\right)$.

The complimentary function of Eq. (28) is $C_{1} I_{1}\left(\Lambda_{n} r\right)+$ $C_{2} K_{1}\left(\Lambda_{n} r\right)$, where $I_{1}\left(\Lambda_{n} r\right)$ is the modified Bessel function of the first kind, and $C_{1}$ and $C_{2}$ are arbitrary constants. Using the method of variation of constants and taking into account the asymptotic relations (Abramowitz \& Stegun 1964)

$I_{1}(x) \sim \frac{\mathrm{e}^{x}}{\sqrt{2 \pi x}}, \quad K_{1}(x) \sim \sqrt{\frac{\pi}{2 x}} \mathrm{e}^{-x}$,

valid for $x \rightarrow \infty$, we obtain that the general solution of Eq. (28) decaying at infinity is given by

$$
\begin{aligned}
F_{n}^{(1)}(r)= & H_{n}^{(1)} K_{1}\left(\Lambda_{n} r\right)+\frac{2 \mathrm{i} \omega_{r} \omega_{i} H_{n}^{(0)}}{V_{\mathrm{Ae}}^{2}} \\
& \times\left\{K_{1}\left(\Lambda_{n} r\right) \int_{R}^{r} s I_{1}\left(\Lambda_{n} s\right) K_{1}\left(\Lambda_{n} s\right) \mathrm{d} s\right. \\
& \left.+I_{1}\left(\Lambda_{n} r\right) \int_{r}^{\infty} s\left[K_{1}\left(\Lambda_{n} s\right)\right]^{2} \mathrm{~d} s\right\} .
\end{aligned}
$$

Here $H_{n}^{(1)}$ is an arbitrary constant. 
Let us calculate the approximate expressions for $P^{(0)}$ and $P^{(1)}$ when $r \sim R$. When doing so we use the formulae (Abramowitz \& Stegun 1964)

$I_{1}(x)=x / 2+O\left(x^{3}\right), \quad K_{1}(x)=x^{-1}+O(x)$,

valid for $0<x \ll 1$. The series (25) and (27) converges very fast, so that $P^{(0)}$ and $P^{(1)}$ can be approximated by a few first terms in (25) and (27) respectively. We will see in what follows that $\omega / V_{\mathrm{Ae}} \sim L^{-1}$. Then it follows from (24) that, for moderate values of $n, \Lambda_{n} \sim L^{-1}$. This implies that, for $r \sim R, \Lambda_{n} r \sim R / L \ll 1$. Now, using the second equation in (31), we obtain from (25)

$$
\begin{aligned}
& P^{(0)}=r^{-1} A_{\mathrm{e}}^{(0)}(z)\left[1+O\left((R / L)^{2}\right)\right], \\
& A_{\mathrm{e}}^{(0)}(z)=\sum_{n=1}^{\infty}\left(H_{n}^{(0)} / \Lambda_{n}\right) G_{n}(z) .
\end{aligned}
$$

Using (31), we obtain for $r \sim R$

$$
\int_{R}^{r} s I_{1}\left(\Lambda_{n} s\right) K_{1}\left(\Lambda_{n} s\right) \mathrm{d} s \approx \frac{r^{2}-R^{2}}{4} .
$$

Since $s\left[K_{1}\left(\Lambda_{n} s\right)\right]^{2}$ has a non-integrable singularity at $s=0$ and exponentially decays as $s \rightarrow \infty$, the main contribution in the integral $\int_{r}^{\infty} s\left[K_{1}\left(\Lambda_{n} s\right)\right]^{2} \mathrm{~d} s$ comes from the vicinity of $r$ when $r \sim R$. Then, taking $R \ll r_{0} \ll L$ and using (31) we obtain

$\int_{r}^{\infty} s\left[K_{1}\left(\Lambda_{n} s\right)\right]^{2} \mathrm{~d} s \approx \int_{r}^{r_{0}} \frac{\mathrm{d} s}{\Lambda_{n}^{2} s}=\Lambda_{n}^{-2} \ln \frac{r_{0}}{r}$.

Now it follows from (31), (33) and (34) that the second term on the right-hand side of (30) is approximately equal to

$$
\frac{\mathrm{i} \omega_{r} \omega_{i} H_{n}^{(0)}}{\Lambda_{n} V_{\mathrm{Ae}}^{2}}\left(\frac{r^{2}-R^{2}}{2 r}+r \ln \frac{r_{0}}{r}\right) \sim \frac{r H_{n}^{(0)}}{\Lambda_{n} L^{2}} \sim \frac{(R / L) H_{n}^{(0)}}{L \Lambda_{n}}
$$

for $r \sim R$. When deriving this estimate we have used $\omega_{r} / V_{\mathrm{Ae}} \sim$ $\omega_{i} / V_{\mathrm{Ae}} \sim L^{-1}$ and $\ln \left(r_{0} / r\right) \sim 1$. Then, using (27), (31), (32) and (35), we obtain from (30)

$P^{(1)}=r^{-1} A_{\mathrm{e}}^{(1)}(z)\left[1+O\left((R / L)^{2}\right)\right]+\widetilde{P}^{(1)}$,

where

$A_{\mathrm{e}}^{(1)}(z)=\sum_{n=1}^{\infty}\left(H_{n}^{(1)} / \Lambda_{n}\right) G_{n}(z), \quad \widetilde{P}^{(1)} \sim(R / L)^{2} P^{(0)}$.

Using (32) and (36) and neglecting terms of the order $(R / L)^{2}$ and $(l / R)^{2}$, we eventually arrive at

$$
\begin{aligned}
& P=P^{(0)}+(l / R) P^{(1)}=r^{-1} A_{\mathrm{e}}(z), \\
& A_{\mathrm{e}}(z)=A_{\mathrm{e}}^{(0)}(z)+(l / R) A_{\mathrm{e}}^{(1)}(z) .
\end{aligned}
$$

We would like to emphasize that the obtained solution in the external region is only valid under the assumption $l \ll R$. The method of variable separation can be also used in the case of arbitrary $l$. In that case we arrive at the same boundary value problem (24) for function $G(z)$, however with $\omega$ substituted for $\omega_{r}$. Since $\omega$ is complex, Eq. (24) has complex coefficients, so that we do not obtain the classical Sturm-Liouville problem. As a result, to obtain a complete system of functions, we have to take not only the eigenfunctions, but also the associate functions (Naimark 1967), which makes the analysis much more involved.

\section{Connection formulae}

The concept of connection formulae was first introduced by Sakirai et al. (1991), and then further developed by Goossens et al. (1995, see also Goossens \& Ruderman 1995). The concept of connection formulae can be described as follows. As we have already stated, in weakly dissipative plasmas dissipation is only important in a thin dissipative layer embracing the ideal resonant surface. Outside this layer the ideal MHD equations can be used. Inside the dissipative layer all equilibrium quantities can be approximated by the first terms of Taylor expansions near the resonant position. As a result the linear dissipative MHD equations are simplified to the form that can be solved analytically. Using the analytical solution we can obtain the connection formulae, which are the expressions for the jumps of the normal velocity component and the total pressure perturbation across the dissipative layer. After that we can consider the dissipative layer as a surface of discontinuity, solve the linear ideal MHD equations at the two sides of the dissipative layer, and then use the connection formula to connect the solutions across the discontinuity. This approach is similar to the use of the Rankine-Hugoniot relations at shocks for studying the motions of ideal compressible fluids.

The original connection formulae were derived for onedimensional equilibria, where, in cylindrical geometry, all equilibrium quantities vary in the radial direction only. However, the generalization for stratified magnetic tubes is straightforward. In this section we briefly describe this generalization.

The plasma motion in the annulus $R-l<r<R$ is described by Eqs. (6), (7) and (9). Let us introduce the functions $U=\partial(r u) / \partial r$ and $\Psi=P / \rho$, and expand $u, U, P$ and $\Psi$ in the generalized Fourier series similar to (13). The characteristic scale of variation of the equilibrium quantities in the radial direction is equal to $l$, while the characteristic scale for perturbations in a dissipative layer is much smaller than $l$. Then, recalling that $W_{n}$ is independent of $r$, we obtain the following expressions

$$
\begin{gathered}
\frac{\partial^{2} u}{\partial r^{2}}=\sum_{n=1}^{\infty} W_{n} \frac{\mathrm{d}^{2} u_{n}}{\mathrm{~d} r^{2}}, \quad \frac{\partial^{2} U}{\partial r^{2}}=\sum_{n=1}^{\infty} W_{n} \frac{\mathrm{d}^{2} U_{n}}{\mathrm{~d} r^{2}}, \\
\frac{1}{\rho} \frac{\partial P}{\partial r} \approx \frac{\partial \Psi}{\partial r}=\sum_{n=1}^{\infty} W_{n} \frac{\mathrm{d} \Psi_{n}}{\mathrm{~d} r} .
\end{gathered}
$$

Substituting the expansions for $u, U, P$ and $\Psi$ in Eqs. (6) and (9), and using Eqs. (12) and (39), we obtain

$\left(\omega^{2}-\lambda_{n}\right) u_{n}-\mathrm{i} \nu \omega \frac{\mathrm{d}^{2} u_{n}}{\mathrm{~d} r^{2}}=-\mathrm{i} \omega \frac{\mathrm{d} \Psi_{n}}{\mathrm{~d} r}$,

$\left(\omega^{2}-\lambda_{n}\right) U_{n}-\mathrm{i} v \omega \frac{\mathrm{d}^{2} U_{n}}{\mathrm{~d} r^{2}}=\frac{\mathrm{i} r \omega}{\rho V_{\mathrm{A}}^{2}}\left(\omega^{2}-\lambda_{n}\right) P_{n}-\frac{\mathrm{i} \omega}{r} \Psi_{n}$,

where $n=1,2, \ldots$ The $n$th term in the expansion of $u$ (and also $U$ ) in the generalized Fourier series is resonant only if there is $r_{n}$ such that $\lambda_{n}\left(r_{n}\right)=\omega_{r}^{2}$, where $\omega_{r}$ is the real part of $\omega$. In that case the equation $r=r_{n}$ defines the ideal resonant surface, and the dissipative layer embraces this surface. The thickness of the dissipative layer is much smaller than $l$, which enables us to use the approximation

$\omega_{r}^{2}-\lambda_{n}(r)=\Delta_{n}\left(r-r_{n}\right), \quad \Delta_{n}=-\left.\frac{\mathrm{d} \lambda_{n}}{\mathrm{~d} r}\right|_{r=r_{n}}$.

The characteristic scale of variation of perturbations in the dissipative layer is defined by the balance of the two terms on the left 
hand side of Eqs. (40) or (41). When $\omega$ is real, we immediately obtain that this characteristic scale is equal to $l_{\mathrm{d}}=\left(v l / \omega_{r}\right)^{1 / 3}$, and the thickness of the dissipative layer is of the order $l_{\mathrm{d}}$. It is obvious that these estimates remain valid also when $\omega$ is complex, $\omega=\omega_{r}-\mathrm{i} \gamma$, if the decrement $\gamma$ satisfies the estimate $\gamma \lesssim l_{\mathrm{d}}\left|\Delta_{n}\right| / \omega_{r} \sim\left(l_{\mathrm{d}} / l\right) \omega_{r}$. This estimate is valid only if $l / R \lesssim l_{\mathrm{d}} / l$. However, for typical conditions in the solar corona $l / R \gg l_{\mathrm{d}} / l$. In that case $\gamma \sim(l / R) \omega_{r}$ for a non-stratified magnetic tube (e.g. Ruderman \& Roberts 2002), and we will see in what follows that this estimate remains valid for a stratified tube. Ruderman et al. (1995) showed that the characteristic scale of variation of perturbations in the dissipative layer does not exceed $l_{\mathrm{d}}$ even when $l / R \gg l_{\mathrm{d}} / l$. However, in this case the thickness of the dissipative layer is of the order $l^{2} / R$. Since we assume that $l \ll R$, the thickness of the dissipative layer is still much smaller than $l$.

Continuing our analysis we notice that, taking the thickness of the dissipative layer to be of the order $l^{2} / R$, we obtain that the ratio of the first term on the right-hand side of Eq. (41) to the second term is of the order $\omega_{r}^{2} l R / V_{\mathrm{A}}^{2}$. Taking into account that $\omega_{r} \sim V_{\mathrm{A}} / L$, we obtain that this ratio is of the order $l R / L^{2} \ll 1$. Hence we can neglect the first term on the right-hand side of Eq. (41) in comparison to the second one. Since the characteristic scale of perturbation variation in the dissipative layer is of order $l_{\mathrm{d}}$, it is convenient to introduce the new stretching variable $\sigma=\left(r-r_{n}\right) / l_{\mathrm{d}}$ in the dissipative layer. Then, using Eq. (42), we rewrite Eqs. (40) and (41) in the approximate form:

$$
\begin{aligned}
& \frac{\mathrm{d} \Psi_{n}}{\mathrm{~d} \sigma}=\frac{\Delta_{n} l_{\mathrm{d}}^{2}}{\omega_{r}}\left(\frac{\omega_{r}^{2}}{l \Delta_{n}} \frac{\mathrm{d}^{2} u_{n}}{\mathrm{~d} \sigma^{2}}+\mathrm{i} \sigma u_{n}+\frac{2 \omega_{r} \gamma}{\Delta_{n} l_{\mathrm{d}}} u_{n}\right), \\
& \frac{\mathrm{d}^{2} U_{n}}{d \sigma^{2}}+\left(\frac{\mathrm{i} l \Delta_{n} \sigma}{\omega_{r}^{2}}+\frac{2 \gamma l}{\omega_{r} l_{\mathrm{d}}}\right) U_{n}=\frac{l \Psi_{n}}{R \omega_{r} l_{\mathrm{d}}} .
\end{aligned}
$$

When deriving these equations we have used the approximation $r \approx R$, and the approximation $\omega \approx \omega_{r}$ everywhere except the coefficients at the first terms on the left-hand sides, where we have used $\omega^{2} \approx \omega_{r}^{2}-2 \mathrm{i} \omega_{r} \gamma$.

It follows from Eq. (44) and the estimate $\gamma \sim(l / R) \omega_{r}$ that $U_{n} \sim\left(l \omega_{r}\right)^{-1} \Psi_{n}$. Using this estimate and the relation $U_{n} \approx$ $R \mathrm{~d} u_{n} / \mathrm{d} r$, we obtain

$\frac{\mathrm{d} u_{n}}{\mathrm{~d} \sigma} \sim \frac{l_{\mathrm{d}} \Psi_{n}}{\omega_{r} l R}$

Since the variation of $\sigma$ in the dissipative layer is of the order $l^{2} / R l_{\mathrm{d}}$, Eq. (45) implies that the variation of $u_{n}$ is of the or$\operatorname{der} l\left(\omega_{r} R^{2}\right)^{-1} \Psi_{n}$. On the other hand, it follows from Eqs. (20) and (21) that $u_{n} \sim\left(\omega_{r} R\right)^{-1} \Psi_{n}$ at $r=R-l$. Since $\left(\omega_{r} R\right)^{-1} \gg$ $l\left(\omega_{r} R^{2}\right)^{-1}$, we can use the estimate $u_{n} \sim\left(\omega_{r} R\right)^{-1} \Psi_{n}$ in the dissipative layer. Now, taking into account that the largest term in the brackets on the right-hand side of Eq. (43) is the last one, we obtain the estimate

$\frac{\mathrm{d} \Psi_{n}}{\mathrm{~d} \sigma} \sim \frac{l_{\mathrm{d}} l}{R^{2}} \Psi_{n}$

This equation implies that the variation of $\Psi_{n}$ across the dissipative layers is of the order $(l / R)^{3}\left|\Psi_{n}\right| \ll\left|\Psi_{n}\right|$. Hence, we can neglect this variation and consider $\Psi_{n}$ as a constant inside the dissipative layer. Then it follows that we can neglect the variation of $\Psi$ and $P$ across the dissipative layer. Introducing the jump of function $f(\sigma)$ across the dissipative layer as

$[f]=\lim _{\sigma \rightarrow \infty}\{f(\sigma)-f(-\sigma)\}$ we, in particular, obtain

$[P]=0$.

This is the first connection formula. It is well-known in the theory of resonant waves in one-dimensional equilibria and has a very simple physical meaning. Since the dissipative layer is thin, its inertia is very small and the pressure has to be in balance at the two sides of this layer.

Since $U_{n}=\mathrm{d}\left(r u_{n}\right) / \mathrm{d} r$, we can consider equation (44) as an equation for $r u_{n}$. Then, with $\Psi_{n}$ constant, this equation coincides, with the accuracy up to the notation, with Eq. (8) of Tirry \& Goossens (1996). Using the results obtained by these authors we can immediately write down its solution:

$u_{n}=\frac{\mathrm{i} \omega_{r} \Psi_{n}\left(r_{n}\right)}{R^{2} \Delta_{n}} G(\tau)+$ const.

where

$G(\tau)=\int_{0}^{\infty} \frac{\exp \left(\mathrm{i} x \tau \operatorname{sgn}\left(\Delta_{n}\right)+\Lambda x\right)-1}{x} \mathrm{e}^{-x^{3} / 3} \mathrm{~d} x$,

$\tau=\sigma\left|\frac{l \Delta_{n}}{\omega_{r}^{2}}\right|^{1 / 3}, \quad \Lambda=2 \gamma\left(\frac{\omega_{r}^{2}}{v \Delta_{n}^{2}}\right)^{1 / 3}$.

It is straightforward to obtain $\lim _{\tau \rightarrow \infty}\{G(\tau)-G(-\tau)\}=$ $\pi i \operatorname{sgn}\left(\Delta_{n}\right)$. Then it follows that the jump of $u_{n}$ across the dissipative layer is given by

$\left[u_{n}\right]=-\frac{\pi \omega_{r} \Psi_{n}\left(r_{n}\right)}{R^{2}\left|\Delta_{n}\right|}$

This is the second connection formula. We do not rewrite this formula in terms of $u$ because, in what follows, we use only the expressions for jumps of the Fourier components of $u$.

\section{Variation of velocity and magnetic pressure across the annulus}

In this section we use the connection formulae to calculate the variation of the magnetic pressure and the normal component of the velocity across the annulus. These variations are equal to $\delta P=P(R, z)-P(R-l, z)$ and $\delta u=u(R, z)-u(R-l, z)$ respectively.

We start from calculating $\delta P$. The characteristic scale of variation of perturbations in the $z$-direction is $L$. The characteristic scales of variation of perturbations in the $r$-direction in the internal homogeneous region $(r<R-l)$ and in the annulus $(R-l<r<R)$ are $R$ and $l$ respectively. Using these facts and the estimate $V_{\mathrm{A}} / \omega \sim L$, we obtain from Eq. (9) the estimates for $\delta u$ and for $u$ in the internal homogeneous region,

$\delta u \sim \frac{l L^{2} \omega_{r} P}{\rho R^{2} V_{\mathrm{A}}^{2}}, \quad u \sim \frac{L^{2} \omega_{r} P}{\rho R V_{\mathrm{A}}^{2}}$.

The first of these estimates implies that, in the annulus, $u(r)=$ $\bar{u}[1+O(l / R)]$, where $\bar{u}=u(R-l)$. Then it follows from Eq. (6) with the third term on the left-hand side equal to zero, and Eq. (52) that

$\frac{\partial P}{\partial r}=\frac{\mathrm{i} \rho V_{\mathrm{A}}^{2}}{\omega}\left(\frac{\partial^{2} \bar{u}}{\partial z^{2}}+\frac{\omega^{2}}{V_{\mathrm{A}}^{2}} \bar{u}\right)[1+O(l / R)]$.

Using Eqs. (12), (20) and (21) we reduce Eq. (53) to

$\frac{\partial P}{\partial r}=A_{\mathrm{i}}(z)[1+O(l / R)]$. 
Then, with the aid of the connection formula (48), we obtain from this equation

$\delta P=l A_{\mathrm{i}}(z)[1+O(l / R)]$.

Substituting the expansion of $u$ in the generalized Fourier series in Eq. (9), neglecting the first and second terms in the square brackets on the right-hand side of this equation in comparison with the third one, and recalling that the term on the left-hand side proportional to $v$ is only important in dissipative layers, we obtain

$\left(\lambda_{n}-\omega^{2}\right) \frac{\mathrm{d}\left(r u_{n}\right)}{\mathrm{d} r}=\frac{\mathrm{i} \omega}{r} \Psi_{n}$.

It follows from Eq. (52) that $\delta u \sim(l / R) u$. Since our calculations are only extended to the first order with respect to the small parameter $l / R$, we can retain only the main order terms when calculating $\delta u$. In particular, we can take $\omega \approx \omega_{r}$ and $r \approx R$ in Eq. (56). Let us denote the half-thickness of the dissipative layer as $\epsilon$. Then we obtain from Eq. (56)

$u_{n}= \begin{cases}u_{n}(R-l)+\frac{\mathrm{i} \omega_{r}}{R^{2}} \int_{R-l}^{r} \frac{\Psi_{n}\left(r^{\prime}\right) \mathrm{d} r^{\prime}}{\lambda_{n}\left(r^{\prime}\right)-\omega_{r}^{2}}, & r<r_{n}-\epsilon, \\ u_{n}(R)-\frac{\mathrm{i} \omega_{r}}{R^{2}} \int_{r}^{R} \frac{\Psi_{n}\left(r^{\prime}\right) \mathrm{d} r^{\prime}}{\lambda_{n}\left(r^{\prime}\right)-\omega_{r}^{2}}, & r>r_{n}+\epsilon .\end{cases}$

Using this equation to calculate $u_{n}\left(r_{n}+\epsilon\right)-u_{n}\left(r_{n}-\epsilon\right)$ and taking $\epsilon \rightarrow+0$ yields

$\left[u_{n}\right]=\delta u_{n}-\frac{\mathrm{i} \omega_{0}}{R^{2}} \mathcal{P} \int_{R-l}^{R} \frac{\Psi_{n}(r) \mathrm{d} r}{\lambda_{n}(r)-\omega_{r}^{2}}$,

where $\mathcal{P}$ indicates the principal Cauchy part of an integral. Comparing this expression with Eq. (51) we eventually arrive at

$\delta u_{n}=-\frac{\pi \omega_{r} \Psi_{n}\left(r_{n}\right)}{R^{2}\left|\Delta_{n}\right|}+\frac{\mathrm{i} \omega_{r}}{R^{2}} \mathcal{P} \int_{R-l}^{R} \frac{\Psi_{n}(r) \mathrm{d} r}{\lambda_{n}(r)-\omega_{r}^{2}}$.

This expression was obtained under the assumption that the $n$th term is resonant. If it is not resonant, then there is no dissipative layer for this term and $\left[u_{n}\right]=0$. In addition, there is no singularity in the integrand in the expression for $\delta u_{n}$. As a result this expression is simplified to

$\delta u_{n}=\frac{\mathrm{i} \omega_{r}}{R^{2}} \int_{R-l}^{R} \frac{\Psi_{n}(r) \mathrm{d} r}{\lambda_{n}(r)-\omega_{r}^{2}}$.

The variation of $u$ across the annulus is given by $\delta u(z)=$ $\sum_{n=1}^{\infty} \delta u_{n} W_{n}(z)$.

\section{Matching solutions}

In this section we use the results obtained in the previous section to match the solutions in the internal and external homogeneous regions derived in Sect. 3. As a result we arrive at the equation determining the real part of $\omega$ and the expression for its imaginary part.

First we match the pressure perturbation recalling that $P(R)-$ $P(R-l)=\delta P$. Using Eqs. (20), (38) and (55), and neglecting terms of the order $(l / R)^{2}$, we immediately obtain that $A_{\mathrm{i}}(z)$ and $A_{\mathrm{e}}(z)$ can be expressed in terms of one unknown function $A(z)$ as

$A_{\mathrm{i}}(z)=R^{-1} A(z), \quad A_{\mathrm{e}}(z)=R A(z)$.
Now, with the aid of Eqs. (20) and (59) we rewrite Eq. (6) in the internal homogeneous region as

$\frac{\mathrm{d}^{2} u_{\mathrm{i}}}{\mathrm{d} z^{2}}+\frac{\omega^{2}}{V_{\mathrm{Ai}}^{2}} u_{\mathrm{i}}=-\frac{\mathrm{i} \omega A}{\rho_{\mathrm{i}} V_{\mathrm{Ai}}^{2} R}$

Note that all the quantities in this equation are functions of $z$ only. Substituting $P=(R / r) A(z)$ in Eq. (6) and taking $r \rightarrow R+0$ we obtain equation valid at $r=R$,

$\frac{\mathrm{d}^{2} u_{\mathrm{e}}}{\partial z^{2}}+\frac{\omega^{2}}{V_{\mathrm{Ae}}^{2}} u_{\mathrm{e}}=\frac{\mathrm{i} \omega A}{\rho_{\mathrm{e}} V_{\mathrm{Ae}}^{2} R}$

where $u_{\mathrm{e}}=u(R)$. Using the relation $u_{\mathrm{e}}=u_{\mathrm{i}}+\delta u$ we rewrite this equation as

$\frac{\mathrm{d}^{2} u_{\mathrm{i}}}{\mathrm{d} z^{2}}+\frac{\omega^{2}}{V_{\mathrm{Ae}}^{2}} u_{\mathrm{i}}=\frac{\mathrm{i} \omega A}{\rho_{\mathrm{e}} V_{\mathrm{Ae}}^{2} R}-\frac{\mathrm{d}^{2} \delta u}{\mathrm{~d} z^{2}}-\frac{\omega^{2}}{V_{\mathrm{Ae}}^{2}} \delta u$.

Subtracting Eq. (60) from Eq. (62) yields

$u_{\mathrm{i}}=\frac{1}{\omega^{2}\left(\rho_{\mathrm{i}}-\rho_{\mathrm{e}}\right)}\left(\rho V_{\mathrm{A}}^{2} \frac{\mathrm{d}^{2} \delta u}{\mathrm{~d} z^{2}}+\rho_{\mathrm{e}} \omega^{2} \delta u-\frac{2 \mathrm{i} \omega}{R} A\right)$.

When deriving this equation we have used the fact that $\rho V_{\mathrm{A}}^{2}=$ const. Now we multiply Eqs. (60) and (62) by $V_{\mathrm{Ai}}^{2}$ and $V_{\mathrm{Ae}}^{2}$ respectively and subtract the first one from the second. As a result we obtain

$\frac{\mathrm{d}^{2} u_{\mathrm{i}}}{\mathrm{d} z^{2}}=\frac{1}{\rho_{\mathrm{i}}-\rho_{\mathrm{e}}}\left(\frac{\mathrm{i} \omega\left(\rho_{\mathrm{i}}+\rho_{\mathrm{e}}\right)}{\rho V_{\mathrm{A}}^{2} R} A-\rho_{\mathrm{i}} \frac{\mathrm{d}^{2} \delta u}{\mathrm{~d} z^{2}}-\frac{\rho_{\mathrm{e}} \omega^{2}}{V_{\mathrm{Ai}}^{2}} \delta u\right)$.

Differentiating twice Eq. (63) with respect to $z$ and comparing the result with Eq. (64) we eventually arrive at

$$
\begin{aligned}
\frac{\mathrm{d}^{2} Q}{\mathrm{~d} z^{2}}+\frac{\omega^{2}}{C_{k}^{2}} Q & =-\frac{\mathrm{i} \omega R}{2}\left\{\frac{\mathrm{d}^{2}}{\mathrm{~d} z^{2}}\left(\frac{\rho V_{\mathrm{A}}^{2}}{\omega^{2}\left(\rho_{\mathrm{i}}-\rho_{\mathrm{e}}\right)} \frac{\mathrm{d}^{2} \delta u}{\mathrm{~d} z^{2}}+\frac{\rho_{\mathrm{e}} \delta u}{\rho_{\mathrm{i}}-\rho_{\mathrm{e}}}\right)\right. \\
& \left.+\frac{1}{\rho_{\mathrm{i}}-\rho_{\mathrm{e}}}\left(\rho_{\mathrm{i}} \frac{\mathrm{d}^{2} \delta u}{\mathrm{~d} z^{2}}+\frac{\rho_{\mathrm{e}} \omega^{2}}{V_{\mathrm{Ai}}^{2}} \delta u\right)\right\}
\end{aligned}
$$

where

$Q=\frac{A}{\rho_{\mathrm{i}}-\rho_{\mathrm{e}}}, \quad C_{k}^{2}=\frac{2 B^{2}}{\mu_{0}\left(\rho_{\mathrm{i}}+\rho_{\mathrm{e}}\right)}=\frac{2 V_{\mathrm{Ai}}^{2}}{1+\chi}$.

Since we assume that $\rho(r, z)=f(r) \rho_{\mathrm{i}}(z)$, it follows that $C_{k}=$ $V_{\mathrm{A}}\left(r_{\mathrm{c}}\right)$, where $r_{\mathrm{c}}$ is determined by

$f\left(r_{\mathrm{c}}\right)=\frac{1+\chi}{2}$

Equation (65) is the main equation of this paper. It contains all the information about the oscillation frequencies and decrements as well as eigenfunctions. We solve this equation by means of the regular perturbation method using expansions with respect to the small parameter $l / R$,

$Q=Q_{0}+(l / R) Q_{1}+\ldots, \quad \omega=\omega_{0}+(l / R) \omega_{1}+\ldots$ 


\subsection{Zero order approximation}

Using Eq. (52) it is easy to show that the ratio of the right-hand side of Eq. (65) to its left-hand side is of the order $l / R$. Then, substituting the expansions (68) in Eq. (65), retaining terms of the zero order only, and recalling the boundary conditions (11), we obtain that $Q_{0}$ and $\omega_{0}$ are determined by the Sturm-Liouville problem

$C_{k}^{2} \frac{\mathrm{d}^{2} Q_{0}}{\mathrm{~d} z^{2}}=-\omega_{0}^{2} Q_{0}, \quad Q_{0}( \pm L)=0$.

This result coincides with one obtained by Dymova \& Ruderman (2005) for a magnetic tube with the sharp boundary. It is not surprising at all because in the zero order approximation we neglect terms of the order $l / R$, which is equivalent to neglecting the effect of the transitional layer $R-l<r<R$.

It is interesting to note that, with the accuracy up to notation, the Sturm-Liouville problem (69) is the same as the Sturm-Liouville problem (12), so that everything written about the problem (12) is also valid for the problem (69). In particular, all the eigenvalues of the problem (69) are positive. Since $C_{k}=V_{\mathrm{A}}\left(r_{\mathrm{c}}\right)$, it follows from Eqs. (16) and (66) that the $n$th eigenvalue of the problem (69), $\omega_{0 n}^{2}$, is given by

$\omega_{0 n}^{2}=\frac{\lambda_{n}(R-l)}{f\left(r_{\mathrm{c}}\right)}$

and we can take $Q_{0 n}=q W_{n}$, where $Q_{0 n}$ is the eigenfunction corresponding to the eigenvalue $\omega_{0 n}^{2}$, and $q$ is an arbitrary nonzero constant with the dimension $\mathrm{m}^{7 / 2} \mathrm{~s}^{3}$, which is introduced to have the correct dimension $\mathrm{m}^{2} \mathrm{~s}^{-2}$ for $Q$.

Now we are in a position to prove the a priori assumption made in Sect. 3 that $\Lambda_{1}^{2}>0$. Let us take $\omega_{r}=\omega_{0 n}$ in Eq. (24). Since $\omega_{0 n}^{2}$ is the eigenvalue of the Sturm-Liouville problem (12) with the corresponding eigenfunction $q W_{n}$ when $r=r_{\mathrm{c}}$, we can use Eq. (12) to express $\omega_{0 n}^{2}$ in terms of $W_{n}$,

$\omega_{0 n}^{2}=-\frac{C_{k}^{2}}{W_{n}} \frac{\mathrm{d}^{2} W_{n}}{\mathrm{~d} z^{2}}$

Substituting this expression in Eq. (24) and using the relation $C_{k}^{2} / V_{\mathrm{Ae}}^{2}=\chi / f_{\mathrm{c}}$, where $f_{\mathrm{c}}=f\left(r_{\mathrm{c}}\right)$, we rewrite Eq. (24) as

$W_{n} \frac{\mathrm{d}^{2} G_{1}}{\mathrm{~d} z^{2}}-G_{1} \frac{\chi}{f_{\mathrm{c}}} \frac{\mathrm{d}^{2} W_{n}}{\mathrm{~d} z^{2}}=-\Lambda_{1}^{2} G_{1} W_{n}$,

where $G_{1}$ is the eigenfunction corresponding to the eigenvalue $\Lambda_{1}^{2}$. Dividing by $W_{n}$ and multiplying by $G_{1}$ we can easily reduce this equation to

$\left(1-\frac{\chi}{f_{\mathrm{c}}}\right) G_{1} \frac{\mathrm{d}^{2} G_{1}}{\mathrm{~d} z^{2}}+\frac{\chi G_{1}}{f_{\mathrm{c}} W_{n}} \frac{\mathrm{d}}{\mathrm{d} z}\left[W_{n}^{2} \frac{\mathrm{d}}{\mathrm{d} z}\left(\frac{G_{1}}{W_{n}}\right)\right]=-\Lambda_{1}^{2} G_{1}^{2}$.

The next step is to integrate this identity over the interval $[-L, L]$ and use the integration by parts and the conditions $G_{1}( \pm L)=$ $W_{n}( \pm L)=0$. However, when doing so we encounter one complication. If we consider not the fundamental mode $(n>1)$ then $W_{n}$ has (simple) zeros inside the interval $(-L, L)$, and one of the integrals that we obtain is improper. In what follows we consider the case $n=2$, so that $W_{2}$ has exactly one zero, $z_{1}$, in the interval $(-L, L)$. The generalization for the case $n>2$ is obvious.
Let us take small $\epsilon>0$ and integrate (73) over the interval $[-L, L]$ with the interval $\left[z_{1}-\epsilon, z_{1}+\epsilon\right]$ removed. Then, using integration by parts we obtain

$$
\begin{aligned}
\Lambda_{1}^{2}\left(\int_{-L}^{z_{1}-\epsilon}\right. & \left.+\int_{z_{1}+\epsilon}^{L}\right) G_{1}^{2} \mathrm{~d} z=\left(1-\frac{\chi}{f_{\mathrm{c}}}\right)\left(\int_{-L}^{z_{1}-\epsilon}+\int_{z_{1}+\epsilon}^{L}\right)\left(\frac{\mathrm{d} G_{1}}{\mathrm{~d} z}\right)^{2} \mathrm{~d} z \\
& +\frac{\chi}{f_{\mathrm{c}}}\left(\int_{-L}^{z_{1}-\epsilon}+\int_{z_{1}+\epsilon}^{L}\right) W_{2}^{2}\left[\frac{\mathrm{d}}{\mathrm{d} z}\left(\frac{G_{1}}{W_{2}}\right)\right]^{2} \mathrm{~d} z \\
& -\left.G_{1} \frac{\mathrm{d} G_{1}}{\mathrm{~d} z}\right|_{z_{1}-\epsilon} ^{z_{1}+\epsilon}-\left.\frac{\chi}{f_{\mathrm{c}}} G_{1} W_{2} \frac{\mathrm{d}}{\mathrm{d} z}\left(\frac{G_{1}}{W_{2}}\right)\right|_{z_{1}-\epsilon} ^{z_{1}+\epsilon}
\end{aligned}
$$

The expansion of $W_{2}$ in the Taylor series in the vicinity of $z_{1}$ is $W_{2}=w_{1}\left(z-z_{1}\right)+\ldots$, where $w_{1}$ is a constant. Using the condition that the second term on the left-hand side of Eq. (73) is regular, we can easily show that the corresponding expansion for $G_{1}$ is $G_{1}=g_{0}+g_{2}\left(z-z_{1}\right)^{2}+\ldots$, where $g_{0}$ and $g_{2}$ are constants. Using this expansion to calculate the last term on the right-hand side of Eq. (74), we obtain that this term is equal to $2 \chi g_{0}^{2}\left(\epsilon f_{\mathrm{c}}\right)^{-1}+O(\epsilon)$. Using this result and noticing that the third term on the righthand side of Eq. (74) is of the order $\epsilon$, we rewrite Eq. (73) as

$$
\begin{aligned}
& \Lambda_{1}^{2} \int_{-L}^{L} G_{1}^{2} \mathrm{~d} z=\left(1-\frac{\chi}{f_{\mathrm{c}}}\right) \int_{-L}^{L}\left(\frac{\mathrm{d} G_{1}}{\mathrm{~d} z}\right)^{2} \mathrm{~d} z+\frac{2 \chi g_{0}^{2}}{\epsilon f_{\mathrm{c}}} \\
& +\frac{\chi}{f_{\mathrm{c}}}\left(\int_{-L}^{z_{1}-\epsilon}+\int_{z_{1}+\epsilon}^{L}\right) W_{2}^{2}\left[\frac{\mathrm{d}}{\mathrm{d} z}\left(\frac{G_{1}}{W_{2}}\right)\right]^{2} \mathrm{~d} z+O(\epsilon) .
\end{aligned}
$$

Recalling that $0<\chi<f_{\mathrm{c}}$, we can see that, choosing $\epsilon$ small enough we always can make the right-hand side of Eq. (75) positive, which implies that $\Lambda_{1}^{2}>0$ and there are no leaky modes.

\subsection{First order approximation}

In the first order approximation we collect terms of the order $l / R$ in equation (65). As a result we obtain

$$
\begin{aligned}
\frac{\mathrm{d}^{2} Q_{1}}{\mathrm{~d} z^{2}} & +\frac{\omega_{0}^{2}}{C_{k}^{2}} Q_{1}=-\frac{\mathrm{i} \omega_{0} R^{2}}{2 l}\left\{\frac{\mathrm{d}^{2}}{\mathrm{~d} z^{2}}\left(\frac{\rho V_{\mathrm{A}}^{2}}{\omega_{0}^{2}\left(\rho_{\mathrm{i}}-\rho_{\mathrm{e}}\right)} \frac{\mathrm{d}^{2} \delta u}{\mathrm{~d} z^{2}}+\frac{\rho_{\mathrm{e}} \delta u}{\rho_{\mathrm{i}}-\rho_{\mathrm{e}}}\right)\right. \\
& \left.+\frac{1}{\rho_{\mathrm{i}}-\rho_{\mathrm{e}}}\left(\rho_{\mathrm{i}} \frac{\mathrm{d}^{2} \delta u}{\mathrm{~d} z^{2}}+\frac{\rho_{\mathrm{e}} \omega_{0}^{2}}{V_{\mathrm{Ai}}^{2}} \delta u\right)\right\}-\frac{2 \omega_{0} \omega_{1}}{C_{k}^{2}} Q_{0},
\end{aligned}
$$

Multiplying this equation by $Q_{0}$, integrating by parts, using Eq. (69), the boundary conditions $Q_{0}( \pm L)=Q_{1}( \pm L)=$ $\delta u( \pm L)=0$, and the boundary condition $\mathrm{d}^{2} \delta u / \mathrm{d} z^{2}( \pm L)=0$ that follows from Eqs. (6), (10) and (11), yields

$$
\begin{aligned}
\omega_{1} \int_{-L}^{L} C_{k}^{-2} Q_{0}^{2} \mathrm{~d} z= & -\frac{\mathrm{i} R^{2}}{4 l} \int_{-L}^{L} \frac{Q_{0}}{\rho_{\mathrm{i}}-\rho_{\mathrm{e}}}\left\{\left(\rho_{\mathrm{i}}-\frac{\rho V_{\mathrm{A}}^{2}}{C_{k}^{2}}\right) \frac{\mathrm{d}^{2} \delta u}{\mathrm{~d} z^{2}}\right. \\
& \left.+\omega_{0}^{2}\left(\frac{\rho_{\mathrm{e}}}{V_{\mathrm{Ai}}^{2}}-\frac{\rho_{\mathrm{e}}}{C_{k}^{2}}\right) \delta u\right\} \mathrm{d} z .
\end{aligned}
$$

Now, using the relation $\rho_{\mathrm{e}} / \rho_{\mathrm{i}}=\chi$, Eqs. (66) and (69), and integrating by parts, we simplify Eq. (77) to

$\omega_{1} \int_{-L}^{L} V_{\mathrm{Ai}}^{-2} Q_{0}^{2} \mathrm{~d} z=\frac{\mathrm{i} \omega_{0}^{2} R^{2}(1-\chi)}{8 l(1+\chi)} \int_{-L}^{L} V_{\mathrm{Ai}}^{-2} Q_{0} \delta u \mathrm{~d} z$

Let us consider the $n$th mode of the tube oscillations, so that $\omega_{0}=\omega_{0 n}$. Then $Q_{0}=q W_{n}$. Since $V_{\mathrm{A}}^{2}=V_{\mathrm{Ai}}^{2} / f(r)$, we can substitute $V_{\mathrm{Ai}}^{2}$ for $V_{\mathrm{A}}^{2}$ in Eq. (14). Then, substituting the expansion of 
$\delta u$ in the generalized Fourier series of the form (13) in Eq. (78) and using Eqs. (14) and (17), we rewrite Eq. (78) as

$\omega_{1 n}=\frac{\mathrm{i} \omega_{0} R^{2}(1-\chi)}{8 q l(1+\chi)} \delta u_{n}$

In accordance with Eqs. (16) and (70), $\lambda_{n}\left(r_{\mathrm{c}}\right)=\omega_{0}^{2}$, so that $r_{n}=r_{\mathrm{c}}, \delta u_{n}$ is a resonant term in the expansion of $\delta u$ in the generalized Fourier series, and we have to use the expression (57) for $\delta u_{n}$.

The real part of $\omega_{1}$ gives only small unimportant correction to $\omega_{0}$, so that in what follows we disregard it and take $\omega_{r}=\omega_{0}$. On the other hand, the imaginary part of $\omega_{1}$ is very important because it determines the damping rate of oscillations due to resonant absorption, $\gamma=-(l / R) \mathfrak{J}\left(\omega_{1}\right)$, where $\mathfrak{J}$ indicates the imaginary part of a quantity. Hence, in what follows we concentrate on calculating the imaginary part of $\omega_{1}$.

We start calculation of the decrement of the $n$th mode, $\gamma_{n}$, from evaluating $\Psi_{n}\left(r_{\mathrm{c}}\right)$. In accordance with Eq. (18)

$\Psi_{n}\left(r_{\mathrm{c}}\right)=\int_{-L}^{L} \frac{P\left(r_{\mathrm{c}}, z\right) W_{n}(z)}{\rho\left(r_{\mathrm{c}}, z\right) V_{\mathrm{Ai}}^{2}(z)} \mathrm{d} z$.

Using Eqs. (20) and (59) and neglecting terms of the order $l / R$, we obtain $P\left(r_{\mathrm{c}}, z\right)=A(z)$. Then, with the aid of Eq. (66), we finally arrive at

$P\left(r_{\mathrm{c}}, z\right)=Q_{0}\left(\rho_{\mathrm{i}}-\rho_{\mathrm{e}}\right)=q(1-\chi) \rho_{\mathrm{i}}(z) W_{n}(z)$.

Using Eq. (67) yields

$\rho\left(r_{\mathrm{c}}, z\right)=f\left(r_{\mathrm{c}}\right) \rho_{\mathrm{i}}(z)=\frac{1}{2}(1+\chi) \rho_{\mathrm{i}}(z)$.

Substituting these expressions for $P\left(r_{\mathrm{c}}, z\right)$ and $\rho\left(r_{\mathrm{c}}, z\right)$ in Eq. (80) and using the normalization condition (17) we eventually obtain

$\Psi_{n}\left(r_{\mathrm{c}}\right)=2 q \frac{1-\chi}{1+\chi}$.

Now, using Eqs. (57), (79) and (81), we arrive at the expression for the decrement of the $n$th mode,

$\gamma_{n}=-\frac{\pi \omega_{0 n}^{3}(1-\chi)^{2}}{4 R\left|\Delta_{n}\right|(1+\chi)^{2}}$.

It is worth noting that, although there could be not one but a few resonant terms in the expansion of $u$ in the generalized Fourier series, due to orthogonality condition (14) only the $n$th term contributes in $\gamma_{n}$. It is also interesting that the expression (82) exactly coincides with the expression for the decrement obtained by Ruderman \& Roberts (2002) (see their Eq. (56)) if we notice that in the latter $\rho_{\mathrm{A}}=\frac{1}{2}\left(\rho_{\mathrm{i}}+\rho_{\mathrm{e}}\right)$.

Using the relation $r_{n}=r_{\mathrm{c}}$, and Eqs. (16) and (70) we obtain $\Delta_{n}=\omega_{0 n}^{2} f^{\prime}\left(r_{\mathrm{c}}\right) / f\left(r_{\mathrm{c}}\right)$, where the prime indicates the derivative with respect to $r$. Substituting this expression in Eq. (82), we rewrite it as

$\frac{\gamma_{n}}{\omega_{0 n}}=-\frac{\pi(1-\chi)^{2} f\left(r_{\mathrm{c}}\right)}{4 R(1+\chi)^{2} f^{\prime}\left(r_{\mathrm{c}}\right)}$.

Since $f^{\prime}\left(r_{\mathrm{c}}\right) / f\left(r_{\mathrm{c}}\right) \sim 1 / l, \gamma_{n} / \omega_{0 n} \sim l / R$ as it should be.

\section{Application to coronal loop oscillations}

In this section we consider the application of theoretical results obtained in the previous sections to the coronal loop oscillations. Andries et al. (2005a) concluded on the bases of their numerical

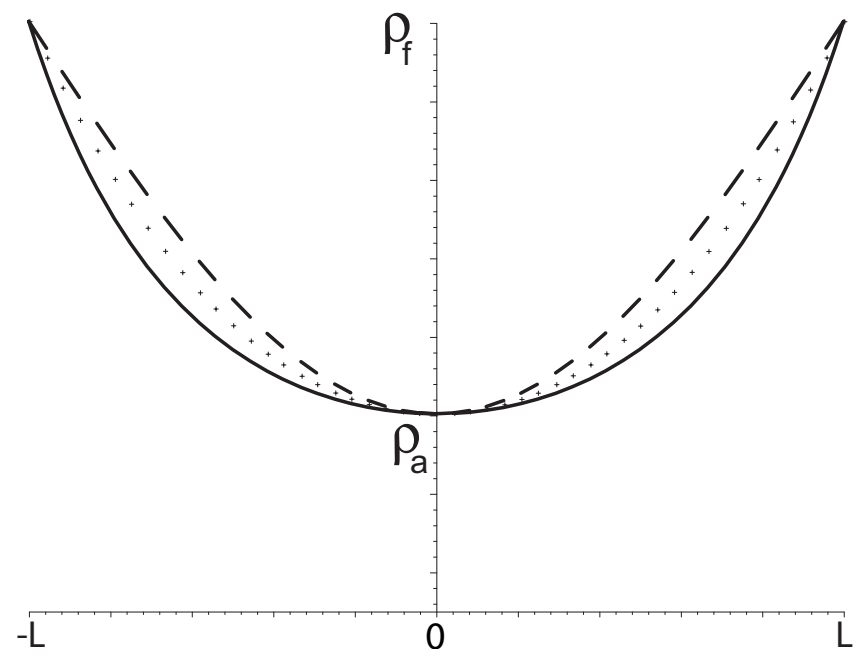

Fig. 2. The density profile inside the loop, $\rho_{\mathrm{i}}(z)$. The solid line shows the density profile given by Eq. (84). The dashed and dotted lines show the sinusoidal density profile used by Andries et al. (2005a), and the density profile in a semicircular loop respectively.

study that the loop oscillation frequency and damping rate are mainly determined by the total mass of the plasma in the loop and the ratio of the densities at the loop apex and at the foot points, $\rho_{\mathrm{a}} / \rho_{\mathrm{f}}$, while the dependence on a particular density distribution inside the loop is weak. Although Andries et al. made their conclusion on the basis of a very restricted set of calculations, their statement looks quite realistic. If we accept this statement, then we can choose $\rho_{\mathrm{i}}(z)$ more or less arbitrarily only imposing the conditions that this function is everywhere convex, i.e. its second derivative is positive, and $\rho_{\mathrm{i}}(-L)=\rho_{\mathrm{i}}(L)$. In what follows we will choose function $\rho_{\mathrm{i}}(z)$ such that we can obtain analytical expressions for the eigenvalues and eigenfunctions of the Sturm-Liouville problem (69). One possible choice is

$\rho_{\mathrm{i}}(z)=\frac{\rho_{\mathrm{a}}}{\left\{1-(1-\kappa)(z / L)^{2}\right\}^{2}}$,

where $\rho_{\mathrm{a}}=\rho_{\mathrm{i}}(0), \rho_{\mathrm{f}}=\rho_{\mathrm{i}}( \pm L)$, and $\kappa=\left(\rho_{\mathrm{a}} / \rho_{\mathrm{f}}\right)^{1 / 2}$. The density profile given by Eq. (84) is shown in Fig. 2 by the solid line. The dashed line shows the density profile used by Andries et al. (2005a), $\rho_{\mathrm{i}}(z)=\rho_{\mathrm{f}}\left[1-\left(1-\kappa^{2}\right) \cos (\pi z / 2 L)\right]$. The dotted line shows the density profile in a semicircular loop embedded in an isothermal atmosphere, $\rho_{\mathrm{i}}(z)=\rho_{\mathrm{f}} \exp [2 \ln \kappa \cos (\pi z / 2 L)]$. We see that the difference between the three profiles is not very large. Hence, we can hope that the eigenvalues and eigenfunctions of the Sturm-Liouville problem (69) are approximately the same for all three density profiles with the same value of $\kappa$ and the same plasma total mass inside the loop.

We also have to give the expression for function $f(r)$ to specify the radial dependence of the density. We choose the same radial density dependence as in Ruderman \& Roberts (2002) and Andries et al. (2005a) and take

$f(r)=\frac{1}{2}\left\{1+\chi-(1-\chi) \sin \frac{\pi(2 r+l-2 R)}{2 l}\right\}$

With the equilibrium density profile given by (84) Eq. (69) takes the form

$\frac{\mathrm{d}^{2} Q_{0}}{\mathrm{~d} z^{2}}+\frac{\mu_{0} \rho_{\mathrm{a}} \omega_{0}^{2}(1+\chi)}{2 B^{2}\left\{1-(1-\kappa)(z / L)^{2}\right\}^{2}} Q_{0}=0$. 


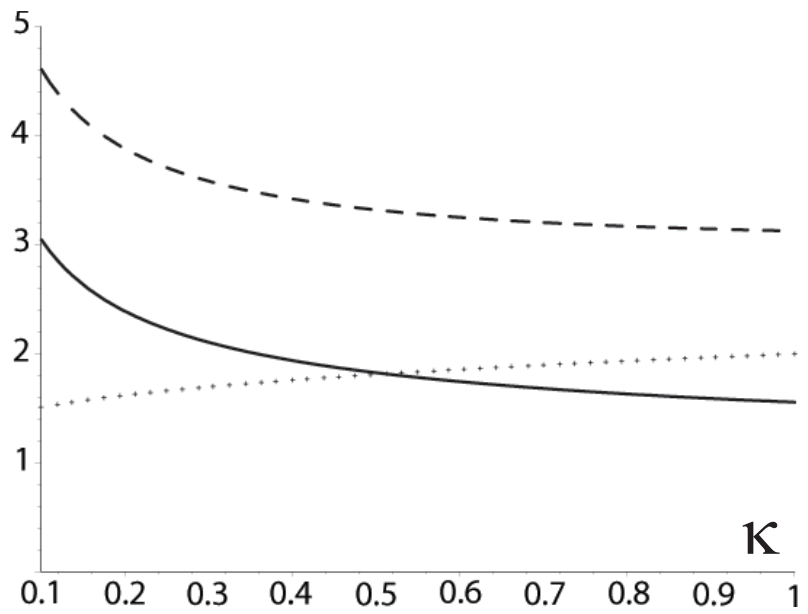

Fig. 3. The solid and dashed lines show the dependences on $\kappa$ of the dimensionless fundamental mode, $\Omega_{n}^{\mathrm{e}}$, and first overtone, $\Omega_{n}^{\mathrm{o}}$, respectively. The dotted line shows the dependence of the ratio of frequencies of the first overtone and fundamental mode, $\omega_{01}^{\mathrm{o}} / \omega_{01}^{\mathrm{e}}$, on $\kappa$.

The general solution of this equation is (see Polyanin \& Zaitsev 2003)

$Q_{0}=\sqrt{1-(1-\kappa)(z / L)^{2}}\left(C_{1} \cos \xi+C_{2} \sin \xi\right)$,

where

$\xi=\alpha \ln \frac{L+z \sqrt{1-\kappa}}{L-z \sqrt{1-\kappa}}, \quad \alpha^{2}=\left(\frac{\mu_{0} \rho_{\mathrm{a}} \omega_{0}^{2} L^{2}(1+\chi)}{8 B^{2}(1-\kappa)}-\frac{1}{4}\right)$,

and $C_{1}$ and $C_{2}$ are arbitrary constants. The boundary conditions $Q_{0}( \pm L)=0$ are satisfied when either $C_{2}=0$ and $\cos \xi_{0}=0$, or $C_{1}=0$ and $\sin \xi_{0}=0$, where $\xi_{0}=\xi(L)$. The first condition corresponds to even modes, and the second to odd modes. Then we obtain that the frequencies of the even modes are given by

$$
\begin{aligned}
& \left(\omega_{0 n}^{\mathrm{e}}\right)^{2}=\frac{\pi B^{2} R^{2} \sqrt{1-\kappa}}{\mu_{0} M L(1+\chi)}\left(\frac{2 \sqrt{1-\kappa}}{\kappa}+\ln \frac{1+\sqrt{1-\kappa}}{1-\sqrt{1-\kappa}}\right) \\
& \quad \times\left\{\pi^{2}(2 n-1)^{2}\left(\ln \frac{1+\sqrt{1-\kappa}}{1-\sqrt{1-\kappa}}\right)^{-2}+1\right\}, n=1,2, \ldots,
\end{aligned}
$$

while the frequencies of the odd modes are given by

$$
\begin{aligned}
\left(\omega_{0 n}^{\mathrm{o}}\right)^{2} & =\frac{\pi B^{2} R^{2} \sqrt{1-\kappa}}{\mu_{0} M L(1+\chi)}\left(\frac{2 \sqrt{1-\kappa}}{\kappa}+\ln \frac{1+\sqrt{1-\kappa}}{1-\sqrt{1-\kappa}}\right) \\
& \times\left\{4 \pi^{2} n^{2}\left(\ln \frac{1+\sqrt{1-\kappa}}{1-\sqrt{1-\kappa}}\right)^{-2}+1\right\}, \quad n=1,2, \ldots,
\end{aligned}
$$

where $M$ is the total mass of the plasma inside the loop,

$$
M=\pi R^{2} L \rho_{\mathrm{a}}\left(\frac{1}{\kappa}+\frac{1}{2 \sqrt{1-\kappa}} \ln \frac{1+\sqrt{1-\kappa}}{1-\sqrt{1-\kappa}}\right)+O(l / R) .
$$

The frequencies of the fundamental mode and the first overtone are given by Eqs. (89) and (90) with $n=1$ respectively. It is straightforward to show that, if $\kappa \rightarrow 1$ (the limit of a non-stratified loop), then Eqs. (89) and (90) give the familiar expression for the frequencies of the even modes, $\omega_{0 n}^{\mathrm{e}}=$ $\pi C_{k}(2 n-1) / 2 L$, and the odd modes, $\omega_{0 n}^{i}=\pi C_{k} n / L$. In Fig. 3 the dependences of the dimensionless frequencies

$\Omega_{n}^{\mathrm{e}, \mathrm{o}}=\frac{\omega_{0 n}^{\mathrm{e}, \mathrm{o}}}{B R} \sqrt{\frac{\mu_{0} M L(1+\chi)}{4 \pi}}$ for the fundamental mode and the first overtone are shown. The ratio of frequencies of the first overtone and fundamental mode is given by

$$
\begin{aligned}
\frac{\omega_{01}^{\mathrm{o}}}{\omega_{01}^{\mathrm{e}}}= & \left\{4 \pi^{2}+\left(\ln \frac{1+\sqrt{1-\kappa}}{1-\sqrt{1-\kappa}}\right)^{2}\right\}^{1 / 2} \\
& \times\left\{\pi^{2}+\left(\ln \frac{1+\sqrt{1-\kappa}}{1-\sqrt{1-\kappa}}\right)^{2}\right\}^{-1 / 2} .
\end{aligned}
$$

The dependence of this quantity on $\kappa$ is shown in Fig. 3.

Using Eqs. (67) and (85), we obtain $r_{\mathrm{c}}=R+l / 2$ and $f^{\prime}\left(r_{\mathrm{c}}\right) / f\left(r_{\mathrm{c}}\right)=l(1+\chi) /(1-\chi)$. Substituting this expression in Eq. (83), we rewrite it as

$\frac{\gamma_{n}}{\omega_{0 n}}=-\frac{\pi l(1-\chi)}{4 R(1+\chi)}$.

With the accuracy up to the notation, this expression is exactly the same as one obtained by Ruderman \& Roberts (2002) for a non-stratified loop with the radial density dependence given by Eq. (85).

We see that the ratio of the damping rate to the oscillation frequency is independent of stratification. The same result was obtained numerically by Andries et al. (2005a). Since this result also follows from a more general expression (83), we conclude that it is valid for any radial and longitudinal dependence of the density. The only essential assumption that this result is based on is the assumption of uniform stratification. Of course, this assumption is only an approximation. In real loops stratifications inside the loop and in the surrounding plasma should be different, mainly because the plasma inside the loop is hotter than the surrounding plasma. However, we believe that this deviation from the uniform stratification is not very strong, so that the conclusion that the ratio of the damping rate to the oscillation frequency is independent of stratification remains approximately valid. Then we can claim that observations of damping of the loop oscillation can provide a very important information about the radial dependence of the density, however they cannot be used to make any conclusions about the density variation along the loops.

To compare our results with those obtained by Andries et al. (2005a) we also solved the Sturm-Liouville problem (69) for the sinusoidal density profile used by Andries et al. In that case the solution of Eq. (69) can be expressed in terms of Mathieu functions. Using this solution we then found the dependence of the fundamental mode frequency on $\kappa$ numerically. Although it was difficult to retrieve accurately the numerical values from the graphical dependence of the fundamental mode frequency on the stratification parameter given by Andries et al., we can guaranty that the difference between our results and the results obtained by Andries et al. does not exceed a couple of percents. This comparison enabled us to claim that our asymptotic solution is very accurate.

One of important reasons for studying coronal loop oscillations is that they can be used for coronal seismology first suggested by Uchida (1970) and Roberts et al. (1984). Nakariakov \& Ofman (2001) used observational data on the periods of loop oscillations to estimate the magnetic field in loops (see also Nakariakov 2001). Ruderman \& Roberts (2002) and Goossens et al. (2002) used the observed damping rate to make conclusions about the density variation in the radial direction. Recently Verwichte et al. (2004) reported simultaneous observation of the fundamental mode and the first overtone in two coronal loops. 
For interpretation of these observations Andries et al. (2005b) used the numerical method described in Andries et al. (2005a) to calculate the ratio of frequencies of the fundamental mode and the first overtone of a semi-circular coronal loop in an isothermal atmosphere. They plotted this ratio as a function of the ratio of the loop radius, $2 L / \pi$ in our notation, to the atmospheric scale $H$ (note that in Andries et al. 2005b the loop length is equal to $L$, and not to $2 L$ as in our paper). If we assume that a loop with the density stratification given by Eq. (84) is in an isothermal atmosphere, then its shape deviates from a half-circle. Therefore, it is convenient to consider the ratio of the apex point height, $z_{a}$, to $H$. For a half-circular loop $z_{a}=2 L / \pi$. The ratio $z_{a} / H$ is related to our parameter $\kappa$ by $z_{a} / H=-2 \ln \kappa$. Andries et al. obtained that the ratio of the frequencies is a monotonically decreasing function of $z_{a} / H$, which agrees very well with our results. We found that the difference between the ratio of frequencies found in our paper and in Andries et al. monotonically grows with $z_{a} / H$. The height of typical coronal loops does not exceed $2 H$. For a loop with the height $2 \mathrm{H}$ the ratio obtained by Andries et al. is 1.67, while our expression (92) gives 1.74. Hence, for $z_{a} / H \leq 2$, the difference between the two ratios of frequencies, one found in our paper and the other obtained by Andries et al., does not exceed 4\%. Only for extremely high coronal loops with the apex point at four scale heights the difference between our result and the result obtained by Andries et al. is fairly large, about $12 \%$.

However, the dependence of $\omega_{01}^{\mathrm{o}} / \omega_{01}^{\mathrm{e}}$ on $\kappa$ is relatively weak. This implies that small variations in $\omega_{01}^{\mathrm{o}} / \omega_{01}^{\mathrm{e}}$ can result in large variations in $\kappa$. For example, for the values reported by Verwichte et al. (2004), which are $\omega_{01}^{\mathrm{o}} / \omega_{01}^{\mathrm{e}}=1.81$ and $z_{a}=70 \mathrm{Mm}$, and $\omega_{01}^{\mathrm{o}} / \omega_{01}^{\mathrm{e}}=1.64$ and $z_{a}=73 \mathrm{Mm}$, the halfcircle model used by Andries et al. (2005b) gives $H=65 \mathrm{Mm}$ and $H=36 \mathrm{Mm}$, while our model gives $H=50 \mathrm{Mm}$ and $H=24 \mathrm{Mm}$. We see that the method of determining the atmospheric scale height using observations of $\omega_{01}^{\mathrm{o}} / \omega_{01}^{\mathrm{e}}$ and the loop height suggested by Andries et al. (2005b) is very sensitive to the stratification law.

\section{Summary and conclusions}

In this paper we have studied transverse oscillations of coronal magnetic loops. We have used the cold plasma approximation, and modelled a loop by a straight magnetic tube with the ends of the magnetic field lines frozen in a dense immovable photosphere. The plasma density varies both along the tube and in the radial direction, the latter variation being confined in a thin annulus near the tube boundary. In addition, we made an assumption of homogeneous stratification, which means that the ratio of densities inside and outside the tube is constant, and the radial dependence of the density in the annulus can be factored out.

In our analysis we have used two small parameters: the ratio of the tube radius to the half-length of the tube, $R / L$, and the ratio of the thickness of the annulus to the tube radius, $l / R$. The accuracy of our calculations with respect to the first parameter is up to terms of the order $R / L$. Hence, we only neglected terms of the order $(R / L)^{2}$. Since, for typical coronal loops, $(R / L)^{2} \lesssim$ $10^{-3}$, the accuracy of our calculations with respect to the first parameter is very high. This conclusion is also supported by the comparison with the numerical solution presented by Andries et al. (2005a).

We have shown that, in the zero order approximation with respect to $l / R$, the real part of the oscillation frequency is an eigenvalue of the Sturm-Liouville problem (69). In the first order approximation with respect to $l / R$, we calculated the decrement (see Eqs. (82) and (83)). The ratio of the decrement and the oscillation frequency is of the order $l / R$. It is interesting to note that the expression for the decrement is exactly the same as in the case of a non-stratified loop, which is a direct consequence of the assumption of homogeneous stratification. This implies that the ratio of the decrement and frequency of the loop oscillation is independent of stratification. Previously this result was obtained numerically by Andries et al. (2005a).

For typical coronal loops $l / R$ can be as large as 0.6 . In addition, we calculated the frequency and decrement of the tube oscillation only in the lowest order approximation with respect to $l / R$. This implies that the accuracy of our results with respect to the parameter $l / R$ is not very high. However, the comparison with the numerical results obtained by Van Doorsselaere et al. (2004a) for non-stratified loops show that the accuracy of our calculations with respect to $l / R$ is quite good for $l / R \leq 0.4$, and it still remains fairly good for $0.4<l / R \leq 0.6$. Hence, our results are accurate enough at least for non-stratified loops. We hope that this conclusion remains valid for stratified loops, although only the comparison with a numerical solution can confirm this assumption.

To apply our results to coronal loop oscillations we have considered a particular stratification when the Sturm-Liouville problem (69) can be solved analytically. This stratification is only slightly different from that in a semicircular loop in an isothermal atmosphere. We calculated the frequencies of the fundamental mode and overtones as functions of the total mass of plasma inside the loop, $M$, the density contrast outside and inside the loop, $\chi$, and the square root from the ratio of densities at the loop apex point and at foot points, $\kappa$. The dependence of any frequency on $M$ and $\chi$ is very simple: it is inversely proportional to $\sqrt{M(1+\chi)}$. The dependences of frequencies of the fundamental mode, $\omega_{01}^{\mathrm{e}}$, and first overtone, $\omega_{01}^{\mathrm{o}}$, on $\kappa$ are shown in Fig. 3. The ratio of this two frequencies is a function of $\kappa$ only, which once again coincides with the result numerically obtained by Andries et al. (2005a).

Andries et al. (2005a) calculated $\omega_{01}^{\mathrm{o}} / \omega_{01}^{\mathrm{e}}$ as a function of the ratio of the loop hight $z_{a}$ to the scale hight $H$, which, for a semicircular loop in an isothermal atmosphere, is given by $2 L / \pi H$. The relation between $z_{a} / H$ and $\kappa$ is $z_{a} / H=-\ln \kappa$. We compared our result with the result obtained by Andries et al. For not very high loops with $z_{a} / H \leq 2$ we obtained that the difference between the two results does not exceed $4 \%$. This result is not very surprising because, in accordance with Fig. 2, the stratification law used in our paper does not differ very much from one used by Andries et al. Further investigation is need to find out how strongly the dependence of $\omega_{01}^{\mathrm{o}} / \omega_{01}^{\mathrm{e}}$ on the ratio of densities at the apex point and the foot points is affected by the variation of the stratification law.

Andries et al. (2005b) used the dependence of $\omega_{01}^{\mathrm{o}} / \omega_{01}^{\mathrm{e}}$ on $z_{a} / H$ for a half-circle loop to calculate the atmospheric scale height on the basis of two simultaneous observations of the fundamental mode and first overtone reported by Verwichte et al. (2004), and obtained $H=65 \mathrm{Mm}$ in the first case, and $H=$ $36 \mathrm{Mm}$ in the second case. We repeated their calculations using the stratification law (84), and obtained $H=50 \mathrm{Mm}$ and $H=24 \mathrm{Mm}$. On the basis of these results we conclude that the method of determining the scale height suggested by Andries et al. (2005b) is very sensitive to the stratification law.

Although the model of a stratified coronal loop used by Andries et al. (2005a) and in this paper is a serious step in the direction of realistic description of coronal loop oscillations, more complicated models should be developed in the future. One of the effects missed in the model of a stratified coronal loop is 
the loop curvature. However, on the basis of the results obtained by Van Doorsselaere et al. (2004b) we do not expect that the account of this effect will sufficiently alter the frequencies and decrements of the loop oscillations.

The second effect worth considering is a non-circular loop cross-section. Ruderman (2003) showed that, for a non-stratified loop with an elliptical cross-section, there are two fundamental modes with different frequencies. There is almost no doubt that the same is true for a stratified loop with an elliptical crosssection.

The third effect that may turn out to be very important is the magnetic field twist inside the loop. Since a strong twist can even destabilize the loop, we can anticipate that a substantial twist that is still below the instability threshold may significantly alter the frequencies of the loop oscillations.

Finally, the effect of nonlinearity comes into play. Very often the observed displacement of the loop axis exceeds its radius. Andries et al. (2005a) argued that "as for the oscillations of a string, the fact that the displacement is much larger than the thickness of the loop/string need not be in disagreement with the assumption of linearity." We can hardly agree with this statement. There is a very big difference between a string and a thin magnetic tube.

The linear theory of a string and a tube is the same, which is clearly seen from Eq. (69). However the nonlinear behaviour of the string is completely different from that of the tube. The phase speed of transverse waves in the string is much smaller than phase speed of sound waves that can propagate in the material the string is made of. This implies that we can consider the string as incompressible when studying its transverse oscillations, and assume that its cross-section does not change. Then we immediately arrive at the conclusion that the linear description of string transverse oscillations is valid as soon as its displacement is much smaller than the wavelength.

In contrast to the string, in general, we cannot neglect the change of the magnetic tube cross-section when studying the tube transverse oscillations. This change of the tube crosssection manifests as the excitation of flute modes. In the long wavelength approximation the phase speeds of all flute modes are the same as the phase speed of the kink mode. In this paper we considered only the kink mode. However Dymova \& Ruderman (2005) considered also the flute modes in a stratified magnetic tube with a sharp boundary. It follows from their analysis that the phase speeds of all flute modes are the same as the phase speed of the kink mode even when the stratification is taken into account. As a result, we can expect that there is resonant nonlinear interaction between the kink mode and the flute modes. Ruderman (1992) studied this problem for a thin homogeneous magnetic tube with a sharp boundary in an incompressible plasma and showed that this resonant interaction does occur. The efficiency of this interaction is characterized by a dimensionless parameter $\epsilon$, which is the ratio of the maximum displacement of the tube axis and the tube radius. The nonlinear effects become important after the time of the order $\epsilon^{-1}$ times the wave period. There is no reason to expect that this estimate is different for a stratified tube in a cold plasma. Then we can state that the nonlinear effects can be neglected only if $\epsilon^{-1}$ times the wave period is larger than the damping time. Since the ratio of the damping time to the wave period is of the order $l / R$, we can write this criterion in the form $\epsilon<l / R$. When $\epsilon \gtrsim 1$ (and oscillations with such a large amplitude are often observed), then the full nonlinear description of the oscillating loop is needed.

Acknowledgements. M.D. acknowledges the support by the University of Sheffield Fellowship and by the Overseas Research Students Award (ORS).

\section{References}

Abramowitz, M., \& Stegun, I. A. 1964, Handbook of mathematical functions (Washington DC: National Bureau of Standards)

Andries, J., Goossens, M., Hollweg, J. V., Arregui, I., \& Van Doorsselaere, T. 2005a, A\&A, 430, 1109

Andries J., Arregui, I., \& Goossens, M. 2005b, ApJ, 624, L57

Aschwanden, M. J., Fletcher, L., Schrijver, C. J., \& Alexander, D. 1999, ApJ, 520,880

Aschwanden, M. J., De Pontieu, B., Schrijver, C. J., \& Title, A. M. 2002, Sol. Phys., 206, 99

Braginskii, S. I. 1965, in Rev. Plasma Phys., 1, 205

Coddington, E. A., \& Levinson, N. 1955, Theory of ordinary differential equations (New York: McGraw-Hill)

Dymova, M., \& Ruderman, M. S. 2005, Sol. Phys., 229, 79

Erdélyi, R., \& Goossens, M. 1995, A\&A, 294, 575

Goossens, M., \& Ruderman, M. S. 1995, Phys. Scri., T60, 171

Goossens, M., Hollweg, J. V., \& Sakurai, T. 1992, Sol. Phys., 138, 233

Goossens, M., Ruderman, M. S., \& Hollweg 1995, Sol. Phys., 157, 75

Goossens, M., Andries, J., \& Aschwanden, M. J. 2002, A\&A, 394, L39

Hollweg, J. V., \& Yang, G. 1988, J. Geophys. Res., 93, 5423

Naimark, M. A. 1967, Linear differential operators, Part I (New York: Frederic Ungar Publ. Co.)

Nakariakov, V. M. 2001, INTAS Workshop on MHD waves in Astrophysical Plasmas, ed. J. L. Ballester, \& B. Roberts (Universitat de les Illes Balears) Nakariakov, V. M., \& Ofman, L. 2001, A\&A, 372, L53

Nakariakov, V. M., Ofman, L., DeLuca, E. E., Roberts, B., \& Davila, J. M. 1999, Science, 285, 862

Ofman, L., Davila, J. M., \& Steinolfson, R. S. 1994, ApJ, 421, 360

Polyanin, A. D., \& Zaitsev, V. F. 2003, Handbook of exact solutions for ordinary differntial equations (Boca Raton: Chapman \& Hall)

Roberts, B. 2000, Sol. Phys., 193, 139

Roberts, B., Edwin, P. M., \& Benz, A. O. 1984, ApJ, 279, 857

Ruderman, M. S. 1992, J. Plasma Phys., 47, 175

Ruderman, M. S. 2003, A\&A, 409, 287

Ruderman, M. S. 2005, ESA SP-600

Ruderman, M. S., \& Roberts, B. 2002, ApJ, 577, 475

Ruderman, M. S., Tirry, W., \& Goossens, M. 1995, J. Plasma Phys., 54, 129

Sakurai, T., Goossens, M., \& Hollweg, J. V. 1991, Sol. Phys., 133, 227

Schrijver, C. J., \& Brown, D. S. 2000, ApJ, 537, L69

Schrijver, C. J., Aschwanden, M. J., \& Title, A. M. 2002, Sol. Phys., 206, 69

Terradas, J., Oliver, R., \& Ballester, J. L. 2006, ApJ, 642, 533

Tirry, W., \& Goossens, M. 1996, ApJ, 471, 501

Titchmarsh, E. C. 1946, Eigenfunction expansions associated with 2nd-order differential equations (Oxford: Oxford Univ. Press)

Uchida, Y. 1970, PASJ, 22, 341

Van Doorsselaere, T., Andries, J., Poedts, S., \& Goossens, M. 2004a, ApJ, 606, 1223

Van Doorsselaere, T., Debosscher, A., Andries, J., \& Poedts, S. 2004b, A\&A, 424, 1065

Verwichte, E., Nakariakov, V. M., Ofman, L., \& DeLuca, E. E. 2004, Sol. Phys., 233, 77 